\title{
Putting water on a lattice: The importance of long wavelength density fluctuations in theories of hydrophobic and interfacial phenomena.
}

\author{
Suriyanarayanan Vaikuntanathan ${ }^{1}$ and Phillip L Geissler ${ }^{1,2}$ \\ ${ }^{1}$ Material Sciences Division, Lawrence Berkeley National Lab, Berkeley, CA 94720. \\ ${ }^{2}$ Department of Chemistry, University of California, Berkeley, CA 94720.
}

\begin{abstract}
The physics of air-water interfaces plays a central role in modern theories of the hydrophobic effect. Implementing these theories, however, has been hampered by the difficulty of addressing fluctuations in the shape of such soft interfaces. We show that this challenge is a fundamental consequence of mapping long wavelength density variations onto discrete degrees of freedom. Drawing from studies of surface roughness in lattice models, we account for the resulting nonlinearities simply but accurately. Simulations show that this approach captures complex solvation behaviors quantitatively.
\end{abstract}

The fluctuating roughness of liquid-vapor interfaces spans a wide range of length scales: from the mesoscopic, where the coarse view of capillary wave theory is appropriate [1], to the microscopic [2,3], where molecular considerations are essential. Computer simulations have demonstrated that these topographical fluctuations can impact an equally broad spectrum of physical responses, with important implications for behaviors of modern interest in biophysics, chemical physics, and materials science including: binding of ligands to hydrophobic protein cavities [4], self assembly of nanoparticles at interfaces [5] and the affinity of diverse solutes for the liquid's boundary [6-8]. Lum, Chandler, and Weeks (LCW) developed a comprehensive conceptual framework for linking such solvation phenomena to fluctuations in the liquid's microscopic density field [9], to which surface roughness clearly contributes. But the corresponding theory has been thoroughly explored only within mean-field approximations for long-wavelength response [9]. Attempts to simultaneously address fluctuations at fine and coarse scales have been hampered by difficulties associated with faithfully representing long-wavelength modes in a statistical mechanics model [10-12] and as a result have suffered from unphysical degeneracies $[10,11]$ or else from the need to introduce numerous parameters that are poorly constrained by available data $[11,12]$. In this Letter we show that previous difficulties in modeling coarse variations in liquid density $[10,11]$ reflect the rich statistical physics of discretely fluctuating surfaces, whose relevance in this context has not been elaborated. In particular, the component of the density field that varies slowly in space is naturally described in numerical approaches by a lattice model. This choice of a discrete representation introduces profound nonlinearities, which can cause decoupling of notionally equivalent measures of surface tension. In extreme cases these nonlinearities can even drive a phase transition from a rough to a quiescent state [14], which lacks the long-wavelength fluctuations altogether.

The basic physics emerging from these nonlinearities has been plumbed in other contexts [14-16]. Here we exploit and extend the resulting understanding and its con-

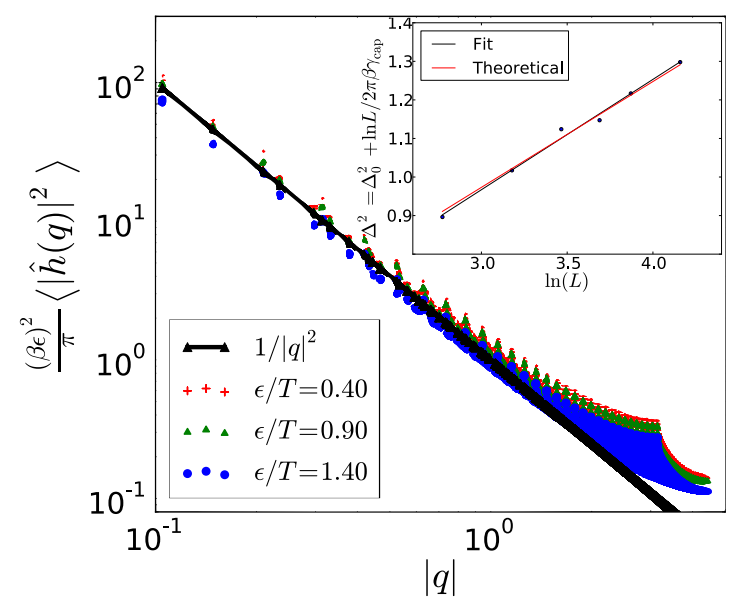

FIG. 1. (Log-Log) Plots of $(\beta \epsilon)^{2}\left\langle|\hat{h}(q)|^{2}\right\rangle / \pi$, where $\hat{h}(q)$ denotes the Fourier modes of interfacial fluctuations in a Solid-on-Solid model. Over a fairly large range of the lattice coupling strength $\epsilon / T$, this plot demonstrates that $(\beta \epsilon)^{2}\left\langle|\hat{h}(q)|^{2}\right\rangle / \pi \approx 1 /|q|^{2}$ for small values of $|q|$, thus verifying the scaling predicted in Eq. 5. Inset: Capillary fluctuations of the lattice gas, at $\epsilon / T=1.35$. The squared interfacial thickness $\Delta^{2}$ determined from simulations, which reflects on the extent of surface roughness, depends logarithmically on the lateral dimension $L$, as predicted by capillary wave theory. The proportionality coefficient for this dependence indicates the surface tension $\gamma_{\text {cap }}$. The value extracted by fitting simulation results to the expected form agrees well with predictions of Eq. 5. Details of calculations in SM [13].

nection with the molecular physics of microscopic density fluctuations. We find that an appreciation of these issues constrains but ultimately simplifies the theoretical task of spanning diverse length scales. The least complicated realization of the LCW perspective, involving no unknown parameters, can in fact suffice to describe quantitatively the solvation of hydrophobic objects with various shapes and sizes (see Fig. 2(a)). This success establishes a minimally complicated model for the hydrophobic effect that is faithful to the intrinsic softness of the air-water interface, and should be useful to investi- 
gate solvation behaviors in various heterogenous environments $[4,12]$. It further allows us to parse contributions from various length scales to material properties of the liquid-vapor interface, such as the Tolman length, bending rigidity, and spontaneous curvature.

The stability of a macroscopic liquid-vapor interface originates in the statistical mechanics of phase transitions. Classic descriptions of long-wavelength variations in that context include phenomenological theories for smooth fields [17] and schematic lattice models that implicitly coarse grain over scales smaller than a lattice spacing $l[1,10]$. The latter involve a minimum of parameters (as few as $l$ and an energy scale $\epsilon$ of microscopic cohesion) and are particularly convenient for numerical simulation. We, like others $[1,10]$, therefore focus on such a discrete representation, where $n_{i}$ indicates the molecular density within lattice cell $i$ in a binary way: $n_{i}=1$ and $n_{i}=0$ denote locally liquid-like and vapor-like density, respectively. Fluctuations of these occupation variables are governed by a lattice gas Hamiltonian,

$$
H=-\epsilon \sum_{\langle i, j\rangle} n_{i} n_{j}-\mu \sum_{i} n_{i},
$$

where $\sum_{\langle i, j\rangle}$ denotes a sum over nearest neighbor cells and $\mu$ is the chemical potential. We have in mind systems like ambient water that are close to coexistence, $\mu \approx$ $-3 \epsilon+\Delta P l^{3}$, where $\Delta P \gtrsim T / l^{3}$ is the difference between ambient pressure and the liquid's vapor pressure, and $T$ is given in units of $k_{\mathrm{B}}$.

How the parameters $l$ and $\epsilon$ should be assigned for a particular material is a surprisingly subtle and pivotal issue. Previous work has argued that $l$ should correspond to the correlation length of density fluctuations in the liquid phase [10]. We are concerned with fluids far below their critical points, setting this length scale slightly in excess of a molecular diameter $(l \approx 4 \AA$ in liquid water). The energy scale $\epsilon$ was inferred from the cost of creating an interface at zero temperature. Equating this cost with the free energy per unit area $\Gamma$ of a real liquid interface at finite temperature yields [10]

$$
\Gamma=\frac{\epsilon}{2 l^{2}} .
$$

According to these arguments, $\epsilon \approx 6.0 T$, in the case of water at ambient conditions. We will show that such a high value is problematic.

While this reasoning is sensible, it neglects entirely the influence of interfacial fluctuations. To account for these shape variations, we consider a macroscopically planar, fluctuating interface between liquid and vapor phases, within the so-called Solid-on-Solid (SOS) limit [1, 18-21]. In this approximation the two phases are each assumed to be internally homogeneous: $n_{i}=1$ everywhere in the liquid phase and $n_{i}=0$ everywhere in vapor, as roughly expected far from criticality. Any configuration of this sort can be specified by the height $h_{i}$ of the liquid phase in each column $i$ of the lattice (taking the interface to be horizontal, with liquid below). At coexistence the Hamiltonian can thus be rewritten as [19]

$$
H_{0}=\frac{\epsilon}{4} \sum_{\langle i, j\rangle}\left|h_{i}-h_{j}\right| .
$$

Our strategy is to estimate the spectrum of capillary waves for the SOS model (at finite temperature), and enforce agreement with that of water. According to capillary wave theory, the Fourier modes $\hat{h}(\mathbf{q})$ of a continuous interface fluctuate with squared amplitude $\left\langle|\hat{h}(\mathbf{q})|^{2}\right\rangle \propto 1 / \gamma_{\text {cap }} q^{2}$, where $\gamma_{\text {cap }}$ denotes the surface tension governing capillary fluctuations. Molecular simulations [11] and experiments [22] indicate that modes of the air-water interface with wavelength $2 \pi / q \gtrsim 1 \mathrm{~nm}$ indeed follow this scaling, and that $\gamma_{\text {cap }}$ corresponds closely with the thermodynamic surface tension $\Gamma[11,23]$. Capillary wave scaling does not necessarily hold for the lattice gas model or the SOS model, whose interfacial roughness depends on temperature in subtle ways [14, 24].

In order to relate $\gamma_{\text {cap }}$ and $\epsilon$ for the SOS model, we ignore for the moment the discrete nature of fluctuations. We examine interfacial statistics of the SOS model by seeking the most representative Gaussian model $H_{\text {cap }}=\frac{\gamma_{\text {cap }}}{4} \sum_{\langle i, j\rangle}\left(h_{i}-h_{j}\right)^{2}$. According to the Gibbs's variational principle,

$$
F_{0} \leq F_{\text {cap }}+\left\langle H_{0}-H_{\text {cap }}\right\rangle_{\text {cap }},
$$

where $\langle\ldots\rangle_{\text {cap }}$ denotes an average taken with respect to the Hamiltonian $H_{\text {cap }}, F_{\text {cap }}$ denotes the free energy corresponding to $H_{\text {cap }}$, and $F_{0}$ denotes the free energy corresponding to the SOS Hamiltonian $H_{0}$. This bound yields an optimal parameterization (see SM [13] for derivation)

$$
\beta \gamma_{\text {cap }}=\frac{(\beta \epsilon)^{2}}{\pi},
$$

where $\beta=1 / T$. This result, scaling quadratically with $\epsilon$, is clearly distinct from the low-temperature relationship in Eq. 2. We performed numerical simulations of the SOS model with values of $\epsilon / T$ in the range $0.4 \leq \epsilon / T \leq 1.6$ and found the variational estimate to be very accurate for $\epsilon / T \lesssim 1.4$ (see Fig. 1 and SM [13]). Comparison with the lattice gas is favorable over a more limited range, since the SOS approximation breaks down at low values of $\epsilon$ as the critical point $\epsilon_{c} / T \approx 0.89$ is approached. Specifically, the range over which the capillary surface tension predicted by Eq. 5 mirrors that of the lattice gas is roughly bounded on the lower end by $\epsilon \gtrsim 1.25 T$. We obtain this lower bound by computing $\gamma_{\text {cap }}$ for the lattice gas model at coexistence [25] and comparing these estimates to those predicted by Eq. 5. The details of the calculation are presented in the SM [13]. We will argue that the range, $1.25 \lesssim \epsilon / T \lesssim 1.40$, is ideal for representing liquid-vapor interfaces. 
The breakdown of our variational estimate at high values of $\epsilon / T$ reflects a well-known singularity in the statistical physics of discretely fluctuating surfaces [14, 15]. Above a critical value, $\epsilon_{\mathrm{R}} / T \approx 1.63$, roughness of the SOS surface is markedly suppressed [14]. This transition to a quiescent interface, which lacks capillary wave scaling, is generic to models that feature a minimum energetic penalty for local deviations from flatness [14, 15]. Our variational estimate breaks down for values of $\epsilon / T$ noticeably below the roughening value. For values of $\epsilon / T$ below but close to the roughening transition, the discrete constraints on lattice fluctuations, which we have ignored while deriving Eq. 5, become relevant and $\beta \gamma_{\text {cap }} \neq$ $(\beta \epsilon)^{2} /(\pi)$. Their effects can approximately be assessed by adding a potential $V_{0} \equiv-2 y_{0} \epsilon \sum_{i} \cos \left(2 \pi h_{i}\right)$ to the SOS Hamiltonian, Eq. 3, with continuous height fluctuations. This potential penalizes configurations in which height fluctuations deviate from discrete values [15] with the constant $y_{0}$ determining the strength of this penalty. Standard methods $[15,16]$ can be used to calculate the corresponding renormalized surface tension, $\gamma_{\text {cap }}$. We choose $y_{0}$ so that the value of $\epsilon / T$ at which this modified system undergoes a roughening transition is close to that of the SOS lattice. We then find that for $\epsilon / T \lesssim 1.4$, there is no significant renormalization due to the discrete constraints. These calculations are described in the SM [13].

Based on this analysis we argue that the range of lattice gas parameters consistent with the physics of hydrophobic solvation is quite narrow. Large hydrophobic objects induce local drying, generating microscopic analogs of a macroscopic interface between liquid and vapor. Faithfully capturing fluctuations of such microscopic interfaces requires that $\epsilon$ be smaller than the critical value for roughening of the lattice gas interface, $\epsilon<\epsilon_{\mathrm{R}}$ [14]. For values of $\epsilon$ slightly below $\epsilon_{\mathrm{R}}$, the discrete nature of the lattice does not entirely suppress long-wavelength capillary modes, but it nonetheless significantly influences the statistics of surface fluctuations. For example, the surface tension of the lattice gas is anisotropic in this regime [24], depending on the orientation of the interface with respect to the axes of the cubic lattice. The implications of this and related lattice artifacts for solvation behaviors of convex objects on cubic lattices have been discussed previously $[11,26]$. They encourage using lattice coupling energies that are weaker still, $\epsilon / T \lesssim 1.4$, for which discreteness is an unimportant feature.

The SOS approximation, which relies upon spatial uniformity within each phase, is well motivated for liquid water at ambient conditions. Here, and in most liquids near their triple points, spontaneous density fluctuations away from the average bulk value $\rho_{l}$ are typically small even on molecular length scales. The range of cohesive energies $1.25 T \lesssim \epsilon \lesssim 1.4 T$ for which the variational estimate in Eq. 5 is faithful is hence also optimal to represent fluctuations in real, far-from-critical liquids. Through Eq. 5, this range of cohesive energies implies a correspondingly narrow range of appropriate lattice spacing $l$. In the case of water, using the experimental value of surface tension [11], $\gamma_{\text {cap }} \approx 17.4 T / \mathrm{nm}^{2}=\epsilon^{2} /\left(\pi l^{2}\right)$, this coarse graining length should, according to our arguments, lie between 1.7 and $1.9 \AA$. Some previous work has adopted values of $l$ close to this range [27], but in each case has assigned an energy scale through the low-temperature relationship Eq. 2.

Having tightly constrained the possible choices of $\epsilon$ and $l$, we focus on implications for the theory and modeling of aqueous solutions: Can a suitably parameterized lattice model for long wavelength variations in density, together with a simple theory for molecular scale fluctuations, accurately predict nontrivial solvation behavior? To do so, we employ the LCW perspective in its simplest incarnation, put forth in Ref [9]. Locally liquid-like regions $\left(n_{i}=1\right)$ additionally support short-wavelength density fluctuations which are assumed to obey Gaussian statistics [2], as characterized by the two-point correlation function $\chi\left(\mathbf{r}-\mathbf{r}^{\prime}\right)=\rho_{l} l\left(\mathbf{r}-\mathbf{r}^{\prime}\right)+\rho_{l}^{2}\left(g\left(\mathbf{r}-\mathbf{r}^{\prime}\right)-1\right)$, where $g(r)$ denotes the radial distribution function [17].

We consider solutes that are ideally hydrophobic, whose sole influence is to exclude solvent from a volume $v$. The constraint of solvent evacuation within a lattice cell $i$ can be accommodated either through variation in the slowly varying density field $\left(n_{i}=0\right)$, or else through a variation in the above mentioned short wavelength Gaussian density field in a locally liquid-like region. Integrating out short-wavelength fluctuations yields an effective Hamiltonian for lattice occupation variables in the presence of a solute $[10,11]$ :

$$
\begin{aligned}
& H_{v}\left[n_{i}\right]=-\epsilon \sum_{\langle i, j\rangle} n_{i} n_{j}-\mu \sum n_{i}+T\left[\frac{N_{v}^{2}}{2 \sigma_{v}}\right]+\frac{T C}{2} \\
& N_{v}=\Sigma_{i} \rho_{l} n_{i} v_{i}, \sigma_{v}=\int_{\mathbf{r} \in v} \int_{\mathbf{r}^{\prime} \in v} \Theta(\mathbf{r}) \chi\left(\mathbf{r}, \mathbf{r}^{\prime}\right) \Theta\left(\mathbf{r}^{\prime}\right)
\end{aligned}
$$

where

$$
C= \begin{cases}\ln \left(2 \pi \sigma_{v}\right) & \text { if }\langle N\rangle_{v}>1, \\ \max \left[\ln \left(2 \pi \sigma_{\mathrm{v}}\right),\langle\mathrm{N}\rangle_{\mathrm{v}}\right] & \text { otherwise, }\end{cases}
$$

and $\Theta(\mathbf{r})=1$ if the lattice cell containing $\mathbf{r}$ is occupied and vanishes otherwise, and $v_{i}$ is the volume of overlap between $v$ and lattice cell $i$. The coarse-grained model defined by Eq. 6 includes as free parameters only the energy and length scales of the underlying lattice gas, which we set as $\epsilon=1.35 T$, and consistent with the statistical mechanics of rough interfaces (Eq. 5), $l=1.84 \AA$ (see inset, Fig. 1). We used $\mu=-3 \epsilon+1.51 \times 10^{-4} T$ [27] for these simulations. Note that the only inputs to this theory are the surface tension of water, and its pair correlation $g(r)[17]$.

Using Monte Carlo simulations of this coarse-grained description, we computed the reversible work $F$ required 


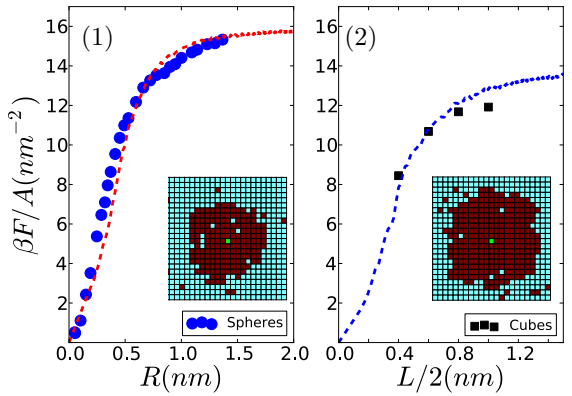

(a)

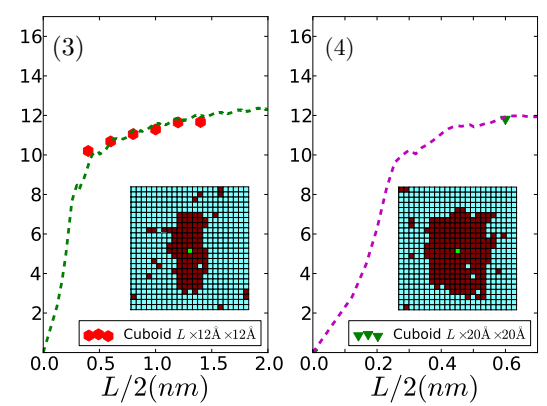

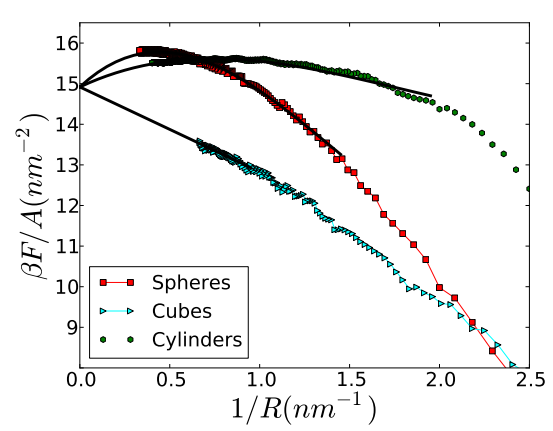

(b)

FIG. 2. (a) Estimates of solvation free energies per unit area of ideal hydrophobic spheres, cubes, and cuboids of various sizes obtained from both the coarse grained lattice model (lines), Eq. 6, and atomistic simulations of SPC/E water (points). (Inset) Cross sections of snapshots of lattice gas simulations using Eq. 6. The slowly varying density field $n_{i}$ is predominantly zero in regions occupied by the solute when the volume of the solute, $v$, is large.(b) Fits (solid lines) of Eq. 9 and Eq. 10 to estimates of solvation free energies per unit area of ideal hydrophobic spheres, and cylinders from the coarse grained lattice model. These fits are used to extract the macroscopic interfacial properties, $\kappa, c_{0}$ and $\delta$ (see text for description). $\Gamma$ is estimated by extrapolating estimates of $F / A$ for cubes from the lattice model to $\lim 1 / L \rightarrow \infty$.

to transfer volume-excluding solutes from vapor into the bulk liquid phase (details of implementation in SM [13]). We focus first on spherical hydrophobes, for which solubility has been previously determined as a function of radius $R$ from detailed molecular simulations [28]. The free energy per unit solute surface area $A=4 \pi R^{2}$ is plotted in Fig. 2(a)(1). Results for the lattice model agree very well with simulation data. As a more stringent test, we computed solvation free energies for hydrophobic objects that vary not only in scale but also in shape. It is not obvious whether a microscopic sphere with given surface area should differ substantially in solubility from, e.g., a cube with the same area. As shown in Fig. 2(a), solvation properties can in fact be very sensitive to such geometric details. For all of the shapes we considered (spheres, cubes, and cuboids), $F / A$ grows rapidly as radius $R$ (or edge length $L$ for the cubes and cuboids considered here) grows to $\sim 1 \mathrm{~nm}$, then increases much more gradually at larger $R$ (or $L$ ). The details of this dependence, however, differ significantly. A naive extrapolation could even suggest that $F / A$ approaches different limiting values at large $R$ for different shapes. These behaviors are observed in molecular simulations (using the SPC/E model of water, details in SM [13]) and for the coarse-grained theory of Eq. 6, with remarkably close correspondence between the two approaches. From the solubility of cubic volumes, we extract the thermodynamic surface tension $\Gamma=14.92 \mathrm{~T} / \mathrm{nm}^{2}$ of our lattice model by extrapolating to $L^{-1} \rightarrow 0$ (see Fig. 2(b)).

The sensitivity of a hydrophobe's solubility to its shape reflects properties of liquid-vapor interfaces that are more subtle than macroscopic surface tension (i.e., $\Gamma$ or $\gamma_{\text {cap }}$ ). Corresponding material parameters are conventionally defined in terms of an expansion in powers of curvature $1 / R$. The Tolman length $\delta$, for example, is defined ac- cording to $F_{s p h} / A=\Gamma(1-2 \delta / R+\ldots)$. A more directly mechanical view is provided by mapping this curvaturedependent response onto that of an elastic sheet. According to Helfrich's phenomenological theory, the elastic free energy of a thin shell with bending rigidity $\kappa$ and spontaneous curvature $c_{0}$ is given by [29]

$$
\begin{aligned}
\frac{F_{s p h}}{A} & =\Gamma-\frac{4 \kappa c_{0}}{R}+C_{1} \frac{1}{R^{2}}+C_{2} \frac{1}{R^{3}}, \\
\frac{F_{c y l}}{A} & =\Gamma-\frac{2 \kappa c_{0}}{R}+\frac{\kappa}{2 R^{2}}+D_{1} \frac{1}{R^{3}},
\end{aligned}
$$

for spherical and cylindrical shapes, respectively. $\left(C_{1}, C_{2}\right.$ and $D_{1}$ are constants determining still more subtle material properties that are not discussed here.) Simultaneously fitting theoretical results for spherical and cylindrical solutes to the form of Eqs. 9,10 (see Fig. 2(b)) yields $\kappa=-3.54 T$ and $c_{0}=0.29 \mathrm{~nm}^{-1}$, and therefore $\delta=2 \kappa c_{0} / \Gamma=-0.14 n m$. These values are consistent with previous estimates from molecular simulation [29].

The ability of such a coarse-grained theory to capture the precise shape dependence of interfacial thermodynamics is striking, given the minimal molecular detail Eq. 6 add to the generic lattice gas. The lattice model, by itself, cannot in fact produce interfaces with nonzero spontaneous curvature $c_{0}$, a consequence of symmetry between liquid and vapor phases in this crude description [30]. Accurately predicted nonzero values of $c_{0}$ and $\delta$ therefore emerge entirely from the coupling of Gaussian density fluctuations at small scales to the generic interfacial roughness of an Ising model. These mechanical details of the air-water interface, which might appear to reflect geometric intricacies of hydrogen bonding, are thus encoded in the simplest measure of microscopic structure in the bulk liquid, its pair correlation function $g(r)$.

We gratefully acknowledge extremely useful discussions with John D Weeks, Gerhard Hummer, David 
Chandler, and David Limmer. This project was supported by the US Department of Energy, Office of Basic Energy Sciences, through the Chemical Sciences Division (CSD) of the Lawrence Berkeley National Laboratory (LBNL), under Contract DE-AC02-05CH11231.

[1] J. D. Weeks, The Journal of Chemical Physics 67, 3106 (1977).

[2] D. Chandler, Physical Review E 48, 2898 (1993).

[3] G. Hummer, S. Garde, A. E. Garcia, A. Pohorille, and L. R. Pratt, Proceedings of the National Academy of Sciences 93, 8951 (1996).

[4] P. Setny, R. Baron, P. Michael Kekenes-Huskey, J. A. McCammon, and J. Dzubiella, Proceedings of the National Academy of Sciences of the United States of America 110, 1197 (2013).

[5] M. Cavallaro, L. Botto, E. P. Lewandowski, M. Wang, and K. J. Stebe, Proceedings of the National Academy of Sciences 108, 20923 (2011).

[6] S. Vaikuntanathan, R. Shaffer, and P. L. Geissler, Faraday Discussions 160, 63 (2013).

[7] D. E. Otten, P. R. Shaffer, P. L. Geissler, and R. J. Saykally, Proceedings of the National Academy of Sciences of the United States of America 109, 701 (2012).

[8] Y. Levin, A. P. Dos Santos, and A. Diehl, Physical Review Letters 103, 257802 (2009).

[9] K. Lum, D. Chandler, and J. D. Weeks, The Journal of Physical Chemistry B 103, 4570 (1999).

[10] P. R. ten Wolde, S. X. Sun, and D. Chandler, Physical Review E 65, 011201 (2001).

[11] P. Varilly, A. J. Patel, and D. Chandler, The Journal of Chemical Physics 134, 074109 (2011).

[12] A. J. Patel, P. Varilly, S. N. Jamadagni, M. F. Hagan, D. Chandler, and S. Garde, The Journal of Physical Chemistry. B 116, 2498 (2012).
[13] See Supplemental Material at [URL will be inserted by publisher] for details of calculation.

[14] J. Weeks, G. Gilmer, and H. Leamy, Physical Review Letters 31, 549 (1973).

[15] S. Chui and J. Weeks, Physical Review Letters 40, 733 (1978).

[16] M. Kardar, Statistical Physics of Fields (Cambridge University Press, 2007).

[17] J. J. S. Rowlinson and B. Widom, Molecular theory of capillarity (Courier Dover Publications, 2002).

[18] H. N. V. Temperley, Mathematical Proceedings of the Cambridge Philosophical Society , 683 (1952).

[19] S. Chui and J. Weeks, Phys. Rev. B 23, 2438 (1981).

[20] M. Fisher, Journal of Statistical Physics 34, 667 (1984).

[21] D. Nelson, T. Piran, and S. Weinberg, Statistical Mechanics of Membranes and Surfaces, Jerusalem Winter School for Theoretical Physics (World Scientific Pub., 2004).

[22] D. G. A. L. Aarts, M. Schmidt, and H. N. W. Lekkerkerker, Science (New York, N.Y.) 304, 847 (2004).

[23] J. Mittal and G. Hummer, Proceedings of the National Academy of Sciences 105, 20130 (2008), http://www.pnas.org/content/105/51/20130.full.pdf+html.

[24] K. K. Mon, S. Wansleben, D. P. Landau, and K. Binder, Physical Review B 39, 7089 (1989).

[25] M. Hasenbusch and K. Pinn, Physica A: Statistical Mechanics and its Applications 192, 342 (1993).

[26] M. Schrader, P. Virnau, D. Winter, T. Zykova-Timan, and K. Binder, The European Physical Journal Special Topics 177, 103 (2009).

[27] A. P. Willard and D. Chandler, Faraday Discussions 141, 209 (2009).

[28] D. M. Huang, P. L. Geissler, and D. Chandler, The Journal of Physical Chemistry B 105, 6704 (2001), http://pubs.acs.org/doi/pdf/10.1021/jp0104029.

[29] F. Sedlmeier and R. R. Netz, The Journal of Chemical Physics 137, 135102 (2012).

[30] M. P. A. Fisher and M. Wortis, Phys. Rev. B 29, 6252 (1984). 


\title{
Supplemental Material for \\ "Putting water on a lattice: The importance of long wavelength density fluctuations in theories of hydrophobic and interfacial phenomena"
}

\author{
Suriyanarayanan Vaikuntanathan ${ }^{1}$ and Phillip L. Geissler ${ }^{1,2,3}$ \\ ${ }^{1}$ Material Sciences Division, Lawrence Berkeley National Lab, Berkeley, CA 94720 \\ ${ }^{2}$ Department of Chemistry, University of California, Berkeley, CA 94720 \\ ${ }^{3}$ Chemical Sciences Division, Lawrence Berkeley National Lab, Berkeley, CA 94720
}

\section{FLUCTUATIONS IN THE SOS MODEL}

The Solid on Solid (SOS) system is described by the Hamiltonian

$$
H_{0}=\frac{\epsilon}{4} \sum_{\langle i, j\rangle}\left|h_{i}-h_{j}\right| .
$$

As described in the main text, we ignore the discrete nature of fluctuations in SOS Hamiltonian above and seek the most representative Gaussian model with Hamiltonian, $H_{\text {cap }}=\frac{\gamma_{\text {cap }}}{4} \sum_{\langle i, j\rangle}\left(h_{i}-h_{j}\right)^{2}$. According to the Gibbs's variational principle,

$$
F_{0} \leq F_{\text {cap }}+\left\langle H_{0}-H_{\text {cap }}\right\rangle_{\text {cap }},
$$

where $\langle\ldots\rangle_{\text {cap }}$ denotes an average taken with respect to the Hamiltonian $H_{\text {cap }}, F_{\text {cap }}$ denotes the free energy corresponding to $H_{\text {cap }}$, and $F_{0}$ denotes the free energy corresponding to the SOS Hamiltonian $H_{0}$ (with continuous fluctuations). We find $\gamma_{\text {cap }}$ by setting

$$
\frac{\partial F_{\text {cap }}}{\partial \gamma_{\text {cap }}}+\frac{\partial}{\partial \gamma_{\text {cap }}}\left\langle H_{0}\right\rangle_{\text {cap }}=0
$$

To proceed, we consider $P_{\text {cap }}(z)$, the probability density associated with observing a height difference $z$ between a particular pair of nearest neighbors, $i$ and $j$, in the Gaussian ensemble,

$$
P_{\text {cap }}(z)=\left\langle\delta\left(h_{i}-h_{j}-z\right)\right\rangle_{\text {cap }}=\frac{1}{2 \pi} \int d k\left\langle e^{-\mathrm{ik}\left(\mathrm{h}_{\mathrm{i}}-\mathrm{h}_{\mathrm{j}}\right)}\right\rangle_{\text {cap }} e^{\mathrm{ikz}} .
$$

To evaluate this density, we set the origin of the two dimensional plane perpendicular to the interface, denoted by $\hat{0}$, at the site $j$ and without loss of generality assume that site $i$ is one lattice unit away on the positive x-axis. Denoting the unit vector along the $\mathrm{x}$-axis by $\hat{x}$, we obtain the following identity (after some tedious but straightforward algebra),

$$
\left\langle e^{-\mathrm{ik}\left(\mathrm{h}_{\hat{\mathbf{x}}}-\mathrm{h}_{\hat{0}}\right)}\right\rangle_{\text {cap }}=\exp \left[\frac{-k^{2}}{2 L^{2}} \sum_{\mathbf{q}} \frac{2-2 \cos (\mathbf{q} \cdot \hat{x})}{\gamma_{\text {cap }}(4-2 \cos (\mathbf{q} \cdot \hat{x})-2 \cos (\mathbf{q} \cdot \hat{y}))}\right],
$$

where $\mathbf{q}$ denotes a (two dimensional) wave vector, $L^{2}$ is the number of lattice sites, and $\sum_{\mathbf{q}}$ denotes a sum over the wave vectors. Using the symmetry of the lattice in $\mathbf{x}$ and $\mathbf{y}$ directions, the summation in Eq. 6 works out to

$$
\sum_{\mathbf{q}} \frac{2-2 \cos (\mathbf{q} \cdot \hat{x})}{(4-2 \cos (\mathbf{q} \cdot \hat{x})-2 \cos (\mathbf{q} \cdot \hat{y}))}=\sum_{\mathbf{q}} \frac{2-2 \cos (\mathbf{q} \cdot \hat{y})}{(4-2 \cos (\mathbf{q} \cdot \hat{x})-2 \cos (\mathbf{q} \cdot \hat{y}))}=\frac{L^{2}}{2}
$$

where we have used the fact that the summation has $L^{2}$ terms. We can now compute the density using Eq. 4 as

$$
P_{\text {cap }}(z)=\sqrt{\beta \gamma_{\text {cap }}} \frac{e^{-\beta \gamma_{\text {cap }} z^{2}}}{\sqrt{\pi}} .
$$

This allows us to compute $\left\langle\left|h_{i}-h_{j}\right|\right\rangle$,

$$
\left\langle\left|h_{i}-h_{j}\right|\right\rangle=2 \int_{0}^{\infty} z P_{\text {cap }}(z) d z=\frac{1}{\sqrt{\pi \beta \gamma_{\text {cap }}}} .
$$


The variational calculation described in Eq. 3 now gives us

$$
\frac{\beta \epsilon}{\sqrt{\pi}\left(\beta \gamma_{\text {cap }}\right)^{3 / 2}}=\frac{1}{\beta \gamma_{\text {cap }}},
$$

which yields

$$
\beta \gamma_{\text {cap }}=\frac{(\beta \epsilon)^{2}}{\pi}
$$

\section{IMPORTANCE OF DISCRETE CONSTRAINTS}

The effects of discrete constraints on Eq. 10 can approximately be assessed by constructing a Sine-Gordon like Hamiltonian [1],

$$
H_{\mathrm{SG}}=\frac{\epsilon}{4} \sum_{\langle i, j\rangle}\left|h_{i}-h_{j}\right|-2 y_{0} \epsilon \sum_{i} \cos \left(2 \pi h_{i}\right),
$$

whose second summation penalizes configurations in which height fluctuations deviate from discrete values. The constant $y_{0}$ determines the strength of this penalty. The renormalized surface tension $\tilde{\gamma}_{\text {cap }}=1 / q^{2}\left\langle|\hat{h}(q)|^{2}\right\rangle_{\mathrm{SG}}$ can be computed using a perturbation theory in $y_{0}$,

$$
\frac{1}{\left\langle|\hat{h}(q)|^{2}\right\rangle_{\mathrm{SG}}}=\frac{1}{\left\langle|\hat{h}(q)|^{2}\right\rangle_{\mathrm{cap}}}-\frac{O\left(y_{0}^{2}\right)}{\left\langle|\hat{h}(q)|^{2}\right\rangle_{\text {cap }}}
$$

where we have mapped the SOS Hamiltonian with continuous fluctuations onto the Gaussian reference Hamiltonian using Eq. 10, observed that the term linear in $y_{0}$ vanishes, and where

$$
O\left(y_{0}^{2}\right)=\frac{\left(2 \epsilon_{0} y_{0}\right)^{2}}{2}\left[\left\langle\sum_{i, j} \cos \left(2 \pi h_{i}\right) \cos \left(2 \pi h_{j}\right)|\hat{h}(q)|^{2}\right\rangle_{\text {cap }}-\left\langle\sum_{i, j} \cos \left(2 \pi h_{i}\right) \cos \left(2 \pi h_{j}\right)\right\rangle_{\text {cap }}\left\langle|\hat{h}(q)|^{2}\right\rangle_{\text {cap }}\right]
$$

Here, $\sum_{i, j}$ denotes a double summation over all lattice sites. After some tedious (but straightforward) algebra [1, 2], Eq. 12 can be rewritten as

$$
\frac{\tilde{\gamma}_{\text {cap }}}{T}=\frac{\gamma_{\text {cap }}}{T}+\left(2 \beta \epsilon y_{0} \pi^{-\pi / \beta \gamma_{\text {cap }}}\right)^{2} \pi^{3} \int_{1}^{\infty} d r(r)^{3-2 \pi / \beta \gamma_{\text {cap }}}
$$

where we have assumed that number of lattice sites, $L^{2}$, is large, replaced the summation $\sum_{i, j}$ by integrals, and have used the approximation

$$
\int_{0}^{\pi r} \frac{1-\mathrm{J}_{0}(q)}{q} \approx \ln \pi r
$$

when $r>>1$. In Eq. 15, $\mathrm{J}_{0}(z)$ denotes the zeroth order Bessel function of the first kind.

This equation can be solved using the Kosterlitz-Thouless renormalization approach [1, 2]. The solutions of the renormalization group flow equations $[1,2]$ are described in Fig. 1 with the definitions $K^{-1} \equiv \gamma_{\text {cap }} / T$, and $y \equiv$ $2 \epsilon y_{0} \pi^{(-\pi K)} / T$. The initial conditions for the flow equations were $K^{-1}=(\beta \epsilon)^{2} / \pi$, and $y_{0}=4.6$. For values $\epsilon / T<1.4$, there is no significant renormalization due to the discrete constraints and $\beta \gamma_{c a p} \approx(\beta \epsilon)^{2} / \pi$. For the value of $y_{0}$ chosen here, the roughening transition is approximately at $\epsilon / T \approx 1.64$. Near this value of $\epsilon / T$, the asymptotic value of the surface tension is equal to theoretically predicted universal value, $\beta \gamma_{\text {cap }}=\pi / 2[1,2]$.

\section{VERIFICATION OF SCALING PREDICTED IN EQ. 10}

As we demonstrated in the main text, the surface tension, $\gamma_{\text {cap }}$, of the SOS lattice is well approximated by the relation in Eq. 10 for values of $\epsilon / T$ approximately below $\epsilon / T \lesssim 1.4$. In Fig. 2, we consider cases $1.5<\epsilon / T \leq 1.6$, which highlights the quantitative importance of lattice discreteness for values of $\epsilon$ even modestly below $\epsilon_{R}$. In particular, even though the SOS lattice supports capillary waves for these values of $\epsilon / T$, its surface tension is markedly different from that predicted by Eq. 10 . 


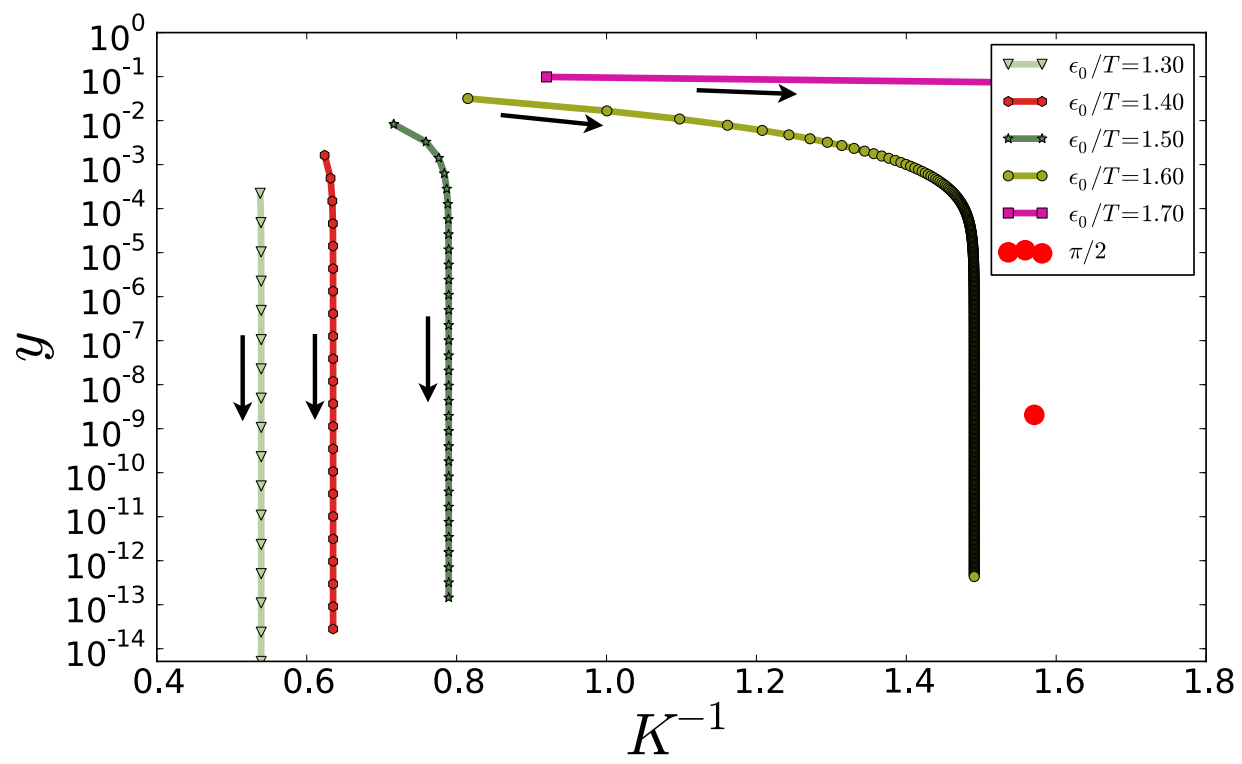

FIG. 1. Evolution of $K^{-1} \equiv \beta \gamma_{\text {cap }}$ and $y$-this reflects the importance of the discrete constraints-under the renormalization flow equations. The arrows indicate the direction of the flow. For $\epsilon / T<1.4, y$ is driven to zero while the surface tension hardly gets renormalized.

\section{ESTIMATES OF SURFACE TENSION OF THE LIQUID VAPOR INTERFACE IN THE LATTICE MODEL}

We computed the capillary surface tension of the lattice gas model at coexistence to verify the scaling predicted in Eq. 10. The interface width was estimated using the technique described in Ref. [3] and requires the use of a clustering algorithm that removes all bubbles from the bulk liquid and all droplets from the vapor phases. The surface tension $\gamma_{\text {cap }}$ is obtained by studying the scaling of the interface width as a function of the lateral dimension, $L$, and using the capillary relation

$$
\Delta^{2}=\Delta_{0}^{2}+\frac{1}{2 \pi \beta \gamma_{\text {cap }}} \ln L,
$$

where $\Delta$ denotes the interface width, and $\Delta_{0}$ is the intrinsic interface width. As we demonstrate in Fig. 3 , Eq. 10 provides an accurate estimate of $\gamma_{\text {cap }}$ only in the range $1.25 \lesssim \epsilon / T \lesssim 1.40$.

\section{IMPLEMENTATION OF THE COARSE GRAINED MODEL}

Implementation of the coarse grained model requires the computation of the integral

$$
\sigma_{v}=\int_{\mathbf{r} \in v} \int_{\mathbf{r}^{\prime} \in v} \Theta(\mathbf{r}) \chi\left(\mathbf{r}, \mathbf{r}^{\prime}\right) \Theta\left(\mathbf{r}^{\prime}\right),
$$

where $\chi\left(\mathbf{r}-\mathbf{r}^{\prime}\right)=\rho_{l} \delta\left(\mathbf{r}-\mathbf{r}^{\prime}\right)+\rho_{l}^{2}\left(g\left(\mathbf{r}-\mathbf{r}^{\prime}\right)-1\right)$, and the other definitions are given in the main text. We approximate Eq. 18 as

$$
\sigma_{v} \approx \sum_{i} \sum_{j} n_{i} b_{v}(i)\left[\int_{\mathbf{r} \in i} \int_{\mathbf{r}^{\prime} \in j} \chi\left(\mathbf{r}, \mathbf{r}^{\prime}\right)\right] n_{j} b_{v}(j),
$$

where $b_{v}(i)$ is the fraction of cell $i$ occupied by the solute. The integral in Eq. 18 is performed using the tables provided in Ref. [4]. 


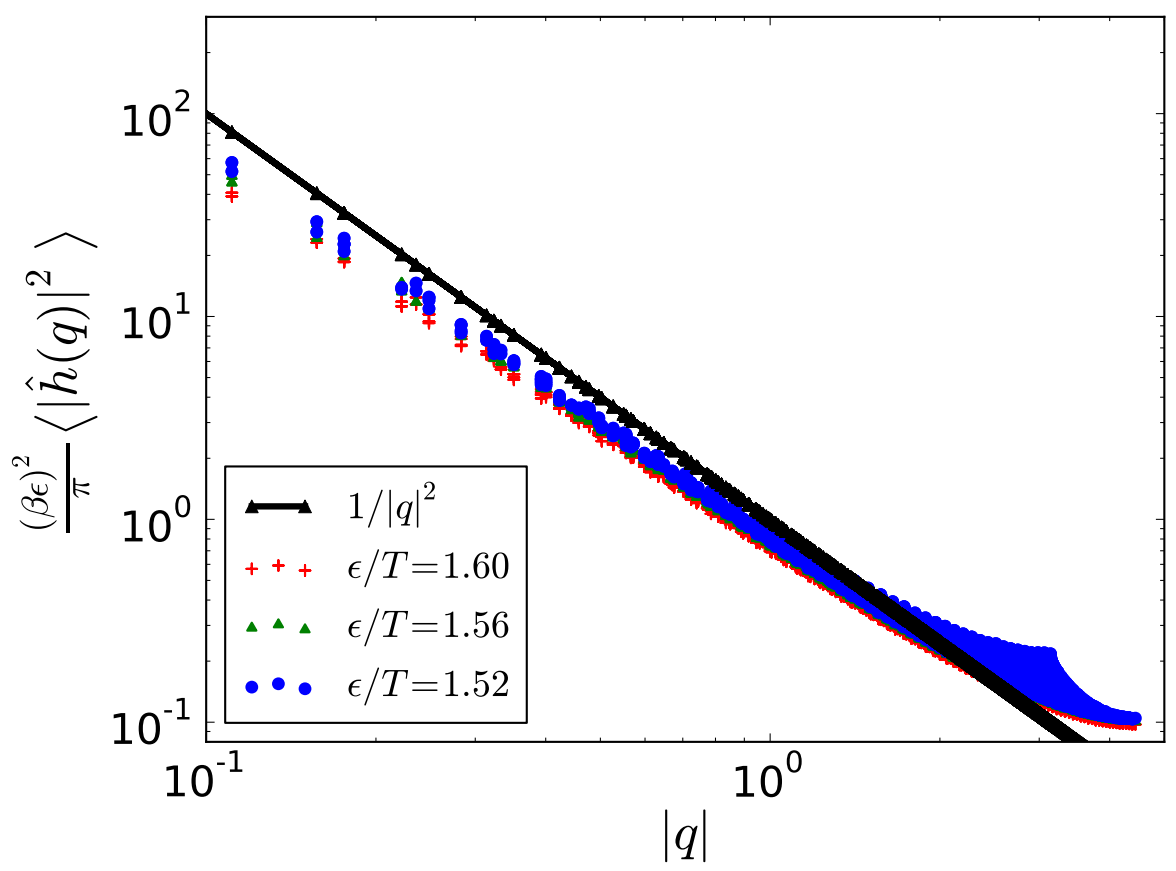

FIG. 2. Breakdown of variational approximation for $\epsilon \leq \epsilon_{R}$. Results for $(\beta \epsilon)^{2}\left\langle|\hat{h}(q)|^{2}\right\rangle / \pi$ from SOS simulations are plotted alongside the prediction $1 /|q|^{2}$ from Eq. 10. For the values of $\epsilon / T$ considered here, the discrete nature of the lattice can no longer be ignored.

\section{DETAILS OF FREE ENERGY CALCULATIONS}

For free energy calculations on the coarse grained model, we solvate volume excluding solutes of various shapes and sizes, interpolating between a point solute and a final size of interest, and sequentially estimate the free energy difference between neighboring solutes in this list using the free energy perturbation identity [5]. We use the Hamiltonian energy function described in the main text for these free energy calculations.

In the case of spheres, we compare free energy estimates from the coarse grained model to estimates of solvation free energy of hard spheres in SPC/E water [6] in Refs. [7, 8]. For the other shapes, we simulated $6912 \mathrm{SPC} / \mathrm{E}$ water molecules in a slab geometry in a NVT ensemble. The dimensions of the slab are $7.2 \mathrm{~nm} \times 7.2 \mathrm{~nm} \times 3.6 \mathrm{~nm}$ with liquid vapor interfaces above and below the slab. The simulation was performed at $T=300 \mathrm{~K}$. Particle Mesh Ewald was used to handle the electrostatic interactions and the SHAKE algorithm was used to constrain the bonds in the water. The simulations were performed using the LAMMPS package. We use the INDUS [9] umbrella sampling method to estimate the solvation free energies. The slab configuration was chosen for reasons described in Ref. [9].

In Fig. 4, we plot estimates of the distribution $P_{v}(N)$, the probability distribution associated with finding $n$ water molecules in a probe volume $v$, obtained from our simulations for $v \sim$ cubes and $v \sim$ cuboids. The solvation free energy is given by $F=-\beta^{-1} \ln P_{v}(0)$.

[1] S. Chui and J. Weeks, Physical Review Letters 40, 733 (1978).

[2] M. Kardar, Statistical Physics of Fields (Cambridge University Press, 2007).

[3] M. Hasenbusch and K. Pinn, Physica A: Statistical Mechanics and its Applications 192, 342 (1993).

[4] A. H. Narten and H. A. Levy, The Journal of Chemical Physics 55, 2263 (1971).

[5] D. Frenkel and B. Smit, Understanding Molecular Simulation, 2nd ed. (Academic Press, San Diego, 2002).

[6] H. J. C. Berendsen, J. R. Grigera, and T. P. Straatsma, The Journal of Physical Chemistry 91, 6269 (1987), http://pubs.acs.org/doi/pdf/10.1021/j100308a038.

[7] D. M. Huang, P. L. Geissler, and D. Chandler, The Journal of Physical Chemistry B 105, 6704 (2001).

[8] D. Chandler, Nature 437 (2005), 10.1038/nature04162. 


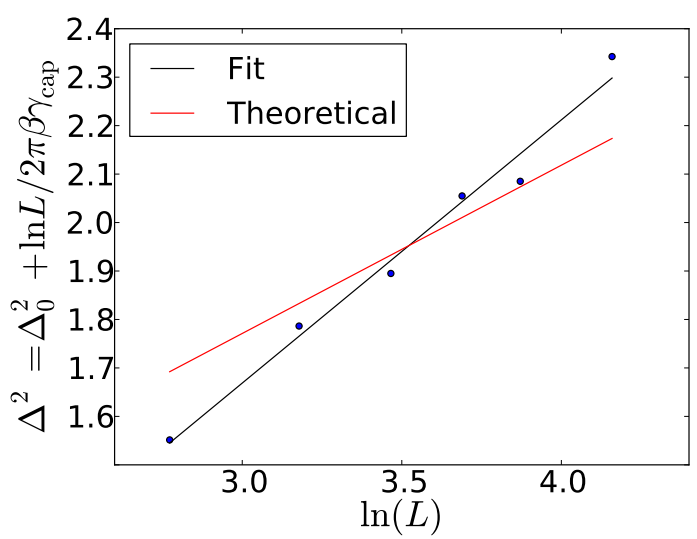

(a) $\epsilon / T=1.20$

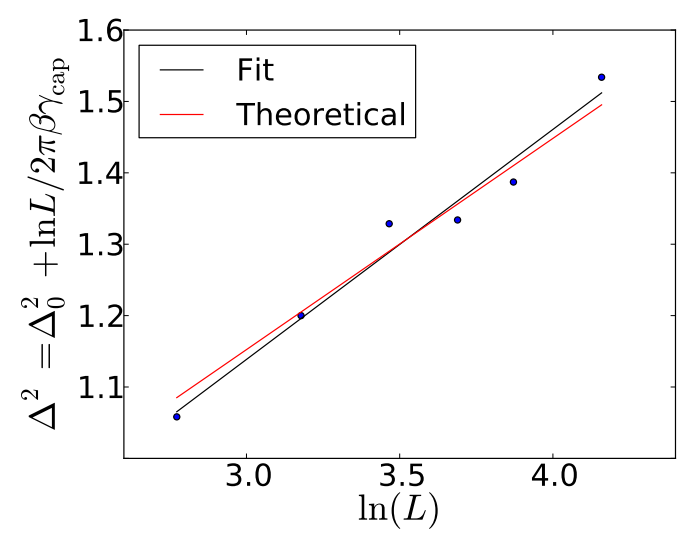

(c) $\epsilon / T=1.30$

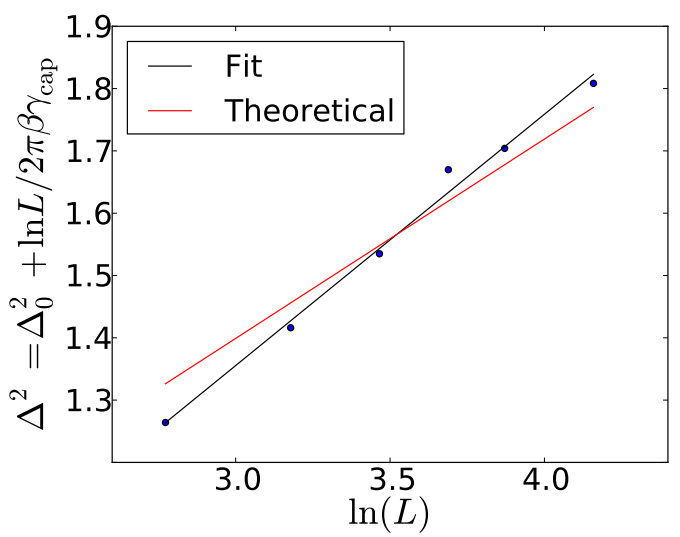

(b) $\epsilon / T=1.25$

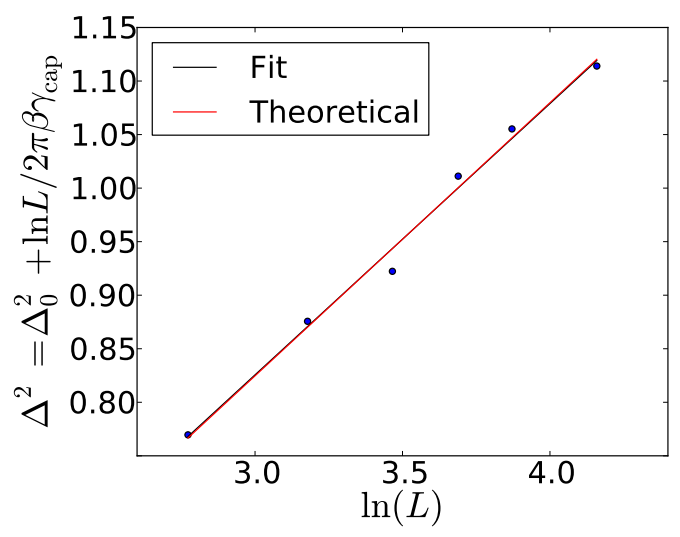

(d) $\epsilon / T=1.40$

FIG. 3. Scaling of the interface width as a function of the lateral dimension $L$ for a lattice gas at coexistence at various values of $\epsilon / T$. Eq. 10 breaks down visibly as $\epsilon / T$ is lowered below $\epsilon / T=1.25$.

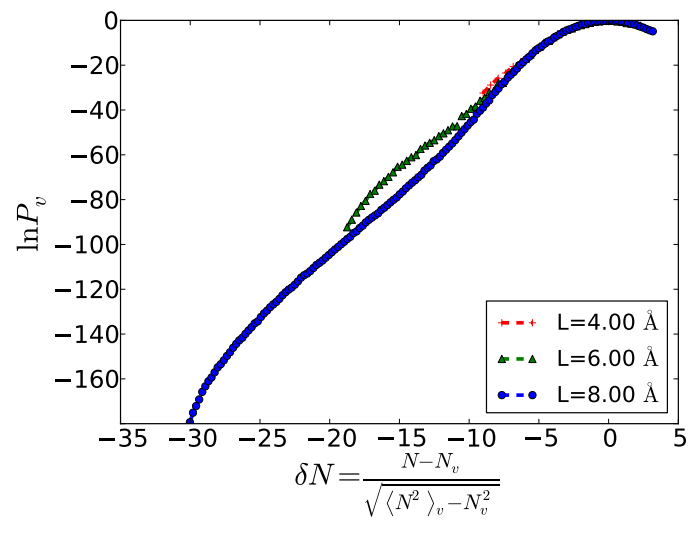

(a)

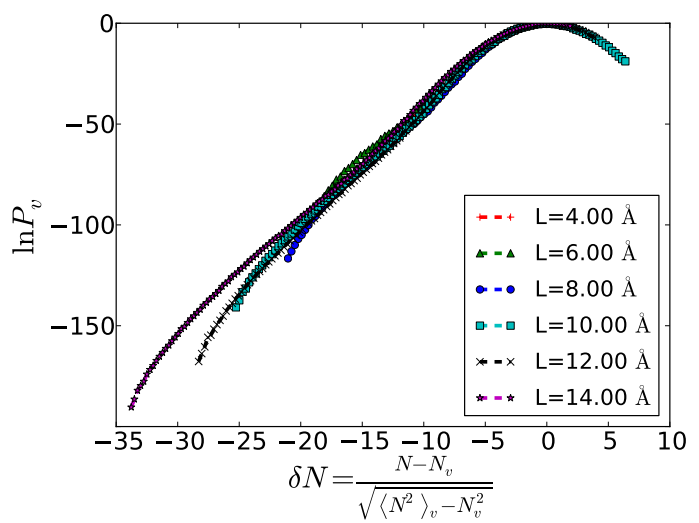

(b)

FIG. 4. Plots of $\ln P_{v}$ as function of $\delta N \equiv \frac{N-N_{v}}{\left\langle N^{2}\right\rangle_{v}-N_{v}^{2}}$, where $N_{v}$ denotes the average number of water molecules in the probe volume $v$. The probe volume $v$ is cuboid shaped $(L \times 12 \AA \times 12 \AA)$ for the instances in Fig. 4(a) and cube shaped for those in Fig. 4(b). See text for details of simulation. 
[9] A. J. Patel, P. Varilly, and D. Chandler, The Journal of Physical Chemistry B 114, 1632 (2010). 